Available online at www.ktu.edu.gh

International Journal of Technology and Management Research 5 (2020)
International Journal

\title{
Four-Wave Mixing Effect and Its Security Implications on a WDM System
}

\author{
Anita Antwiwaa', Seth Okyere-Dankwa², Mensah Sitti and Anil Kumart \\ ${ }^{1}$ Department of ECE, All Nations University, Koforidua, Ghana. Email: draantwiwaa@,anu.edu.gh \\ ${ }^{2}$ Department of Computer Science, Koforidua Technical University, Koforidua, Ghana. \\ Email: Seth.Okyere-dankwa@,ktu.edu.gh \\ 3 Department of Computer Engineering, All Nations University, Koforidua, Ghana \\ Email:msitti@,anu.edu.gh \\ 4Department of ECE,SHUATS, Allahabad, India.Email: Anil.kuman@shuats,edu.in \\ ${ }^{2}$ Correspondence: Seth.Okyere-dankwa@,ktu.edu.gh
}

\begin{abstract}
The data yearning of the world is expanding massively with time and it is convincing media transmission organizations to mount up procedures to satisfy the high information need just as giving a proficient support. During the exponential development of data hungry clients, media transmission organizations fantasize about giving nature of administrations at a lower cost to their clients to endure the challenge in the market. Transmitting products of signs through a solitary direct in perspective on serving a huge number of clients all the while by wavelength division multiplexing (WDM) is the appropriate response. Four-wave multiplexing (FWM) is the optical sign float from one channel to the next. This impact has the inclination of forcing a danger on the fiber correspondence organize consequently undermining the security and secrecy of the data sent by the clients. In this work, FWM impact and its security issues are broken down. The different Tangle lab reenactment consequences of this FPM is contemplated utilizing optical.
\end{abstract}

Keywords: Four-wave mixing, Security, Wavelength division Multiplexing

Citation: Anita Antwiwaa, Seth Okyere-Dankwa, Mensab Sitti and Anil Kumar, Four-Wave Mixing Effect and Its Security

Implications on a WDM System, 2020; 5(4): 1-11.

Received: October 3, 2020

Accepted: December 31, 2020 


\subsection{Introduction}

\subsection{Backeground}

The development of numerous media transmission organizations has required an expanding rivalry among organizations which has prompted giving nature of administration at a lower cost. The lower the expense of administration the higher the interest (client) and the other way around. Accomplishing this point requires a productive technique in which less assets will be utilized to support countless clients simultaneously. The most proficient technique which can help take care of this issue is wavelength division multiplexing (WDM) framework.

WDM is a technology which combines several optical carrier signals onto a single optical fiber by using different wavelengths of laser light (Tkach et al, 1995). This facility helps telecommunication companies to expand their facilities without laying new fibers. As communication technologies advances, security becomes the major heddle to climb. Unauthorized users are working hard to get access to information of users such as credit card details, classified data, and accounts details of customers. These information theft causes billions of losses to the companies every year. WDM as a means of reducing the cost of transmission also has some possible security issues which will be addressed in this work.

\subsection{Security Issues Faced by Some Telecommunication Companies}

Over the years, prominent telecommunication networks have been hacked causing security compromise to customers. Talk'Talk Company was faced with an attack in 2015 which affected the details of about 157,000 of its customers. The intruders accessed 156,959 customers' personal details, 15,656 bank accounts numbers and short codes of these customers were stolen as well. Moreover 28,000 credit and debit card numbers of customers were also stolen. This attack caused the company a loss of $£, 60$ million (Talktalk, 2015).

2000 customers' accounts of Vodafone were hacked in October 2015. The hackers gained the customer's short codes and the last four digits of their accounts. Moreover, they tried to access the bank details with the information gained (Vodafone, 2016).

Indian BSNL website was also attacked in July 2015. This attack caused a compromise of over 30 million records such as customers detail, payments, classified data, etc. (Anonymous, 2016). These attacks affected the companies and the customers adversely therefore there is a need to look for the loopholes and solve it.

The part of nonlinear optics which will be contemplated in this paper is the wonder of association of three optical signal force in the equivalent optical channel. The different dangers brought about by the association of these signs will be considered just as its conceivable security issues.

\subsection{WDM FWM model}

A fiber medium creates some amount of electric field polarization which travels along with the electromagnetic field in the form of polarized waves as the electric field propagates through the medium. Consider a non-linear polarization $P_{N L}$ with a third order susceptibility $\varepsilon_{0} x^{(3)}$

$P_{N L}=\varepsilon_{0} x^{(3)}$

If an optical system generates three frequency signals $\varepsilon_{1}, \varepsilon_{2}, \varepsilon_{3}$ and transmit it through an optical fiber then

$P_{N L}=\varepsilon_{0} x^{(3)}: \varepsilon_{1} \varepsilon_{2} \varepsilon_{3}$ 
The polarization will cause the three signals to interact at frequencies $\mathrm{Fr}_{1}, \mathrm{Fr}_{2}, \mathrm{Fr}_{3}$ producing a fourth frequency $\mathrm{Fr}_{4}$.

$F r_{4}=F r_{1} \pm F r_{2}$

These frequencies will have a wave number $K_{4}=K_{1} \pm K_{2} \pm K_{3}$. The nonlinear nature of the fiber will cause different frequencies to be generated when given as input. These new frequencies will lie near one of the approaching frequencies. At the point when this new sign recurrence falls in the transmission window of the first sign recurrence, extreme crosstalk may happen.

Different signals with Nc number of channels will generate MP number of mixing products which is mathematically given in $(9,10,11)$ as

$M P=\frac{N c^{3}-N c^{2}}{2}$

Equation 4 shows that if there are three signals propagating through a fiber and there is an occurrence of interaction in a four-wave mixing regime, there will be an evolution of nine signal. This means that the interaction will generate six signals (side-bands) in addition to the original three signals. Taking all possible combinations will yield $F r_{1}+F_{2}-F r_{3}$ $, F r_{1}+F r_{3}-F r_{2}, F r_{3}+F r_{2}-F r_{1}, 2 F r_{1}-2 F r_{2}, 2 F r_{1}-2 F r_{3}, 2 F r_{2}-2 F r_{1}, 2 F r_{2}-2 F r_{3}, 2 F r_{3}-2 F r_{1}$ and $2 \mathrm{Fr}_{3}-2 \mathrm{Fr}_{2}$.

with the most damaging signals to system performance given as

$$
F r_{i j k}=F r_{i}+F r_{j}-F r_{k}, \text { wherei, } j \neq k
$$

At the receiving end of a fiber of length $\mathrm{L}$ and attenuation $\alpha$, the power $P_{i j k}$ that is generated at frequency $F r_{i j k}$ due to the signal interaction at frequencies $F r_{i}, F r_{j} F r_{k}$ power input power $P_{i}, P_{j} \wedge P_{k}$ respectively is given by

$P_{i j k}(L)=\eta(g K)^{2} P_{i}(0) P_{j}(0) P_{k}(0) \exp (-\alpha L)$

where $\eta$ is the efficiency of the four-wave mixing, $\boldsymbol{g}$ is the degeneracy factor which has a value of 6 and the non-linear interaction constant $\mathrm{K}$ is given as

$K=\frac{32 \pi^{3} \varepsilon_{0} x^{(3)}}{n_{2} \lambda c}\left(\frac{L_{e f f}}{A_{e f f}}\right)$

where $\varepsilon_{0} x^{(3)}$ is the third-order nonlinear susceptibility, $\mathrm{L}_{\text {eff }}$ is the effective Length, $\mathrm{n}_{2}$ is the refractive index of the fiber and $A_{\text {eff }}$ is the effective cross-sectional area of the fiber. The input signal to the fiber is modeled by the non-linear Schrödinger equation which is given in (12-14) as

$\frac{d A}{d z}+\frac{j}{2} \beta_{2} \frac{d^{2} A}{d T^{2}}+\frac{\alpha}{2} A=j \gamma \vee A \quad{ }^{2} A$

Where $j=-1, A$ is the slowly varying envelope of the optical field, $z$ is the axial distance, $T$ is the time delay $(T=t-z / v g)$, $v g$ is group velocity and $a$ is the fiber loss. The solution to equation 8 for multiple pulse transmission is given as

$A(0, t)=\operatorname{sech}\left(\mu+z_{o}\right)+\operatorname{sech}(\mu)+r \operatorname{sech}\left[r\left(\mu+z_{o}\right)\right] \exp (i \theta)$ 
where $\mu$ is the relative amplitude; $\theta$ is the relative phase, $r$ is the relative amplitude of the pulse and $z_{0}$ is the initial separation between neighboring pulses.

\subsection{Effect of FWM on WDM Systems}

Four-wave mixing (FWM) is the optical signal drift from one channel to the other. This effect has the tendency of imposing a threat on the fiber communication network thereby undermining the security and confidentiality of the information sent by the customers. There are different kinds of attacks that affects an optical network. These attacks depend on the particular network and also the intention and the goal of the attacker. Many authors have classified threats as Service disruption attack which targets on reducing the effectiveness of communication and quality of service (QOS) degradation and tapping attack where the unauthorized users access a data illegally as well as performing traffic analysis on the network thereby affecting the privacy of a communication. Physical layer attack has been classified into direct, indirect and Pseudo attacks.

Equation 9 was simulated for three signals packets at distances $20 \mathrm{~km}$ and $30 \mathrm{~km}$ using MATLAB and the simulation results are given in figure 1 and 2 below.

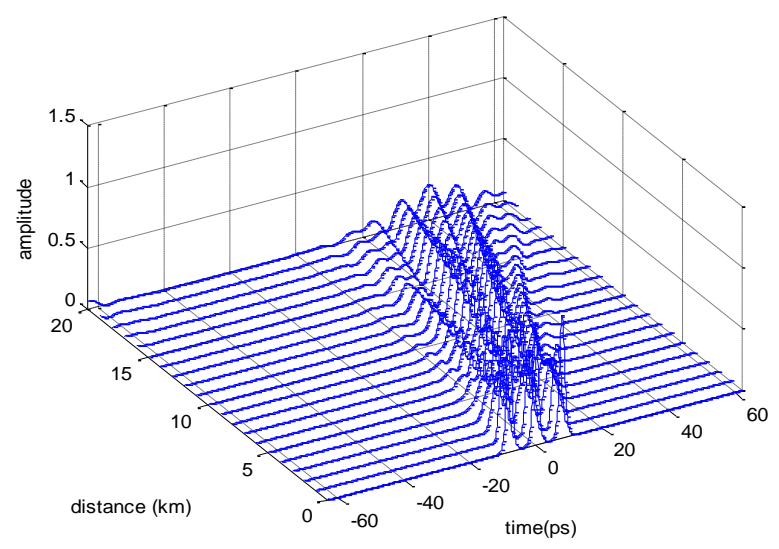

Figure 1 Four-wave mixing of three signals with equal spacing at a distance of $20 \mathrm{Km}$.

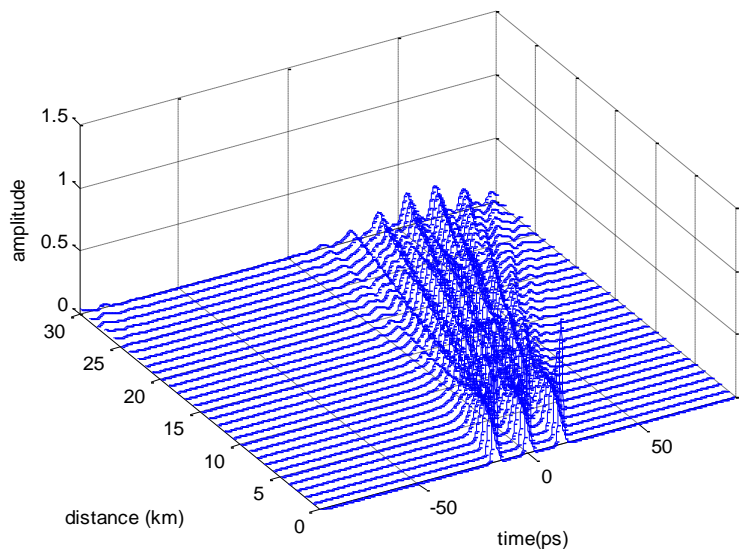

Figure 2 Four-wave mixing of three signals with equal spacing at a distance of $20 \mathrm{Km}$.

Figure 1 and 2 represents three signals transmitted through an optical fiber at a distance of $20 \mathrm{Km}$ and $30 \mathrm{Km}$ respectively. Interaction of the signals in figure 1 commenced from a distance of $2 \mathrm{Km}$ and generated new signal thereby affecting the original power strength. The initial pulse propelled into the fiber was with a sufficiency of around 1 volt however after 
the impact of the information parcel, it very well may be seen that the amplitude of the information bundle has diminished radically to about 0.4 volts which is $60 \%$ abatement in information quality. At separation $30 \mathrm{Km}$ in figure 2 , there is an expansion in wave number from the underlying three to roughly nine. This shows the more extended the separation the more noteworthy the impact of four-wave mixing and the other way around.

\subsection{FWM effect on system security}

As communication technologies advances, security becomes the major hurdle to climb. Unauthorized users are working hard to access information of authorized users' information such as credit card details, classified data and bank details of customers. These actions of the unauthorized users make companies encounter tremendous losses. WDM as a means of reducing the cost of data transmission is liable to security treats due to the four-wave mixing effect (FWM). Figure 4 shows the transmission of three soliton signals $\mathrm{Fr}_{1}, \mathrm{Fr}_{2}, \mathrm{Fr}_{3}$ using a WDM system with equal channel spacing of $\Delta \mathrm{Fr}$ in between them. Occurrence of FWM in the channel will produce a new signal as seen in equation 4. Now considering the intermodulation products from figure 1 yields

$F r_{2}=F r_{1}+2 \Delta F r$

and $F r_{3}=F r_{1}+\Delta F r$

now taking the most affected signal to be

$$
F r_{4}=F r_{1}+F r_{2}-F r_{3}
$$

Substituting equation 10 and 11 into 12 yields

$$
\begin{aligned}
& F r_{4}=F r_{1}+F r_{1}+2 \Delta F r-F r_{1}-\Delta F r \\
& F r_{4}=F r_{1}+\Delta F r
\end{aligned}
$$

equating equation 11 and 13

$$
F r_{4}=F r_{3}: F r_{1}+\Delta F r=F r_{1}+\Delta F r
$$

From equation 15, if $F r_{4}=F r_{3}$ then it means that $F r_{4}$ and $F r_{3}$ carries the same information. This implies that if an eavesdropper or intruder gets access to the FWM newly generated signal $\mathrm{Fr}_{4}$, the security of $\mathrm{Fr}_{3}$ is compromised because the two signals are carrying the same information. Now if channel $F r_{3}$ receives $F r_{1}+\Delta F r$, then some amount of data of $F r_{1}$ would be received by $\mathrm{Fr}_{3}$. This also compromises the security of the signal received. This is shown in the output pulse of figure 1 as blue and red signal mixed together.

Now considering a WDM system $F r_{1}, F r_{2}, F r_{3}$ with unequal channel spacing of $\Delta F r$ and $2 \Delta F r$ between them, their intermodulation products form yields

$F r_{2}=F r_{1}+3 \Delta F r$ 
Substituting equation 16 and 17 into 12 yields

$$
F r_{4}=F r_{1}+F r_{1}+3 \Delta F r-F r_{1}-2 \Delta F r
$$

$F r_{4}=F r_{1}+\Delta F r$

Equating equation 16, 17 and 18 gives

$F r_{4} \neq F r_{3} \neq F r_{2}: F r_{1}+\Delta F r \neq F r_{1}+3 \Delta F r \neq F r_{1}+3 \Delta F r$

Equation 19 shows that when the spacing between the channels are unequal and the intruder is able to access $F r_{4}$ signal then the obtained signal might not contain any useful information because it is not the same as $F r_{1}, \wedge F r_{3}$. This means unequal space channel do well in security than the equal spaced channel. It can also withstand interaction more than the equal space channel. This makes four-wave mixing effect difficult to affect the unequal spaced channel which is shown in figure 3.

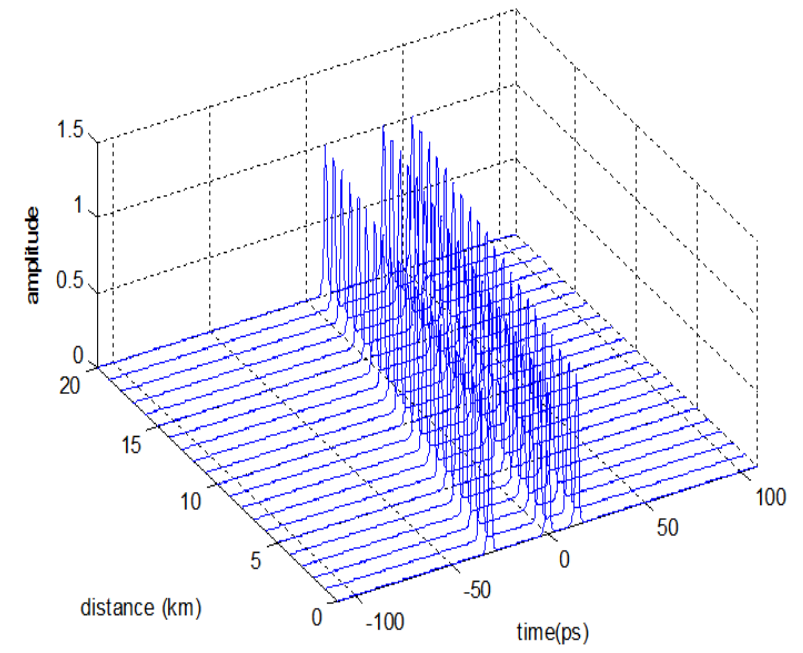

Figure 3: Four-wave mixing of three signals with unequal spacing at a distance of $20 \mathrm{Km}$. 


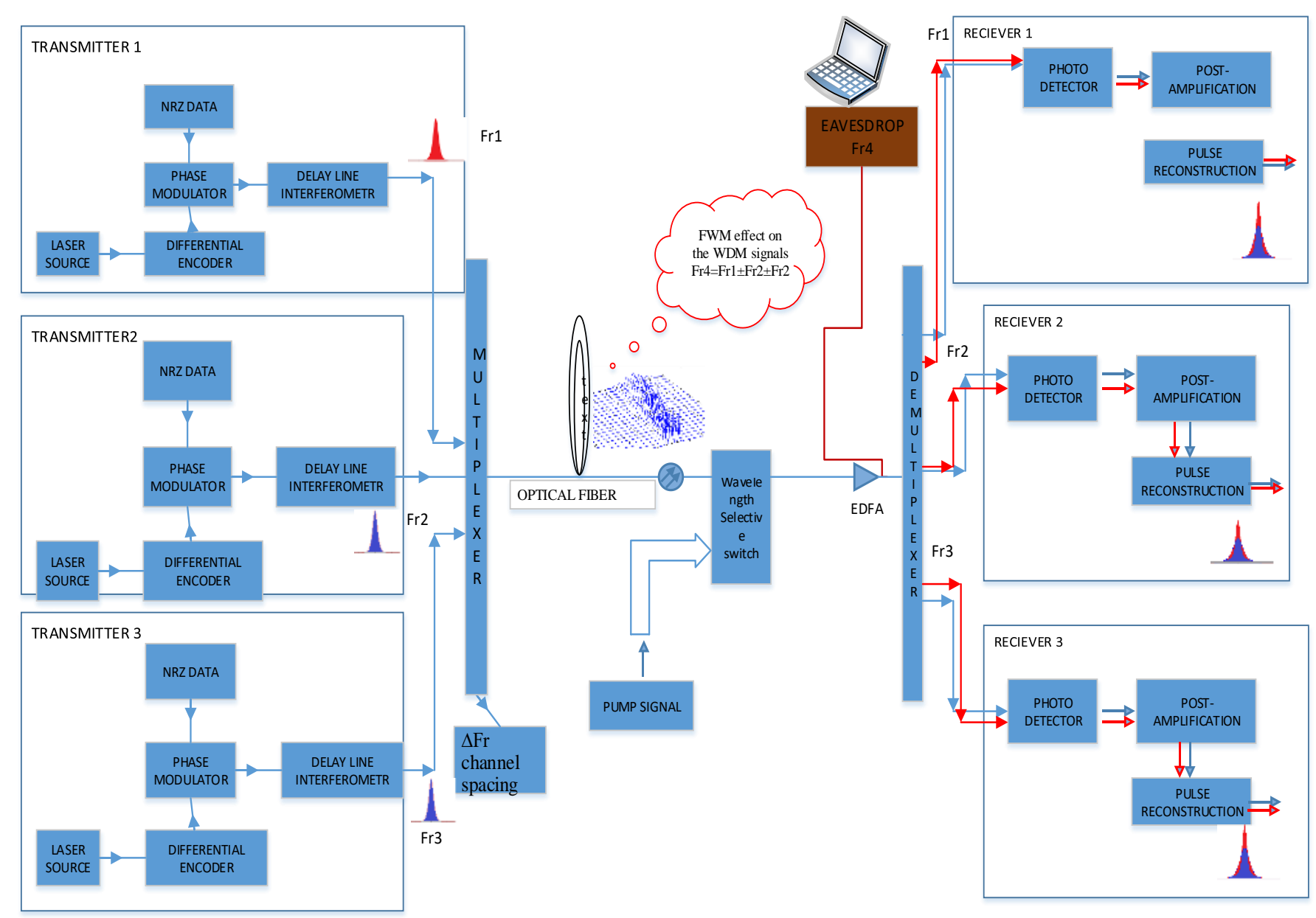

Figure 4: Transmission of three solitons signals using WDM system with FWM effec

\subsection{Secured WDM System using ANITAHALIA SECURITY MODEL}

The issue of security is a major concern for both customers and telecommunication companies. Unauthorized users are finding ways to access information of legitimate users. In this work, we propose a wavelength division multiplexing (WDM) system using ANITHALIA security model with multistage encoding and phase detection. It is essential to protect the actual content of the transmitted data from intruders so that the information will be meaningless to them in case they get access to it. A pseudorandom generated pulse is used instead of a user defined pulse so that it cannot be generated by the intruder since it is random, and they are not repeated. The laser source is photon entangled by splitting into two and changing the polarization state for extra securing of the signal. The photon entangled output is combined and given as input to a Mach Zehnder modulator (MZM) where one arm is phase modulated. Combining the signals from both arms, the phase modulation is converted to intensity modulation. The intensity modulated output is then transmitted unto the optical fiber. The frequencies of the signals are separated using wavelength grating filter. The photo detected out is connected to a phase detector which will compare the phases, separate them, and select the actual phase transmitted. Figure 4 represents a secured WDM system with phase detection. Consider the case in which the intruder mimics the frequency of the legitimate user as shown in figure 6.a and 6.b, it is very difficult to detect such intrusion after multiplexing as given in figure 6.c. Since they are of the same frequency of $193.1 \mathrm{THz}$, the output of that wavelength filter will detect both signals as given in figure 6.d and 6.e represents the signal detected by the $193.2 \mathrm{THz}$ filter. 
In order to separate the intruder's intercepting signal, there is a need for phase detection which will be comparing the output and subtract to get the phase of the actual signal as given in figure 6.f.

Comparing figure 6.b, 6.d and 6.f, the transmitted signal in figure 6.b was not the same as the signal that was reflected in 6.d but the phase detector has been able to extract the legitimate signal resulting in figure 6f. This shows that the ANITHALIA detection method is able to protect the WDM system from intrusion.

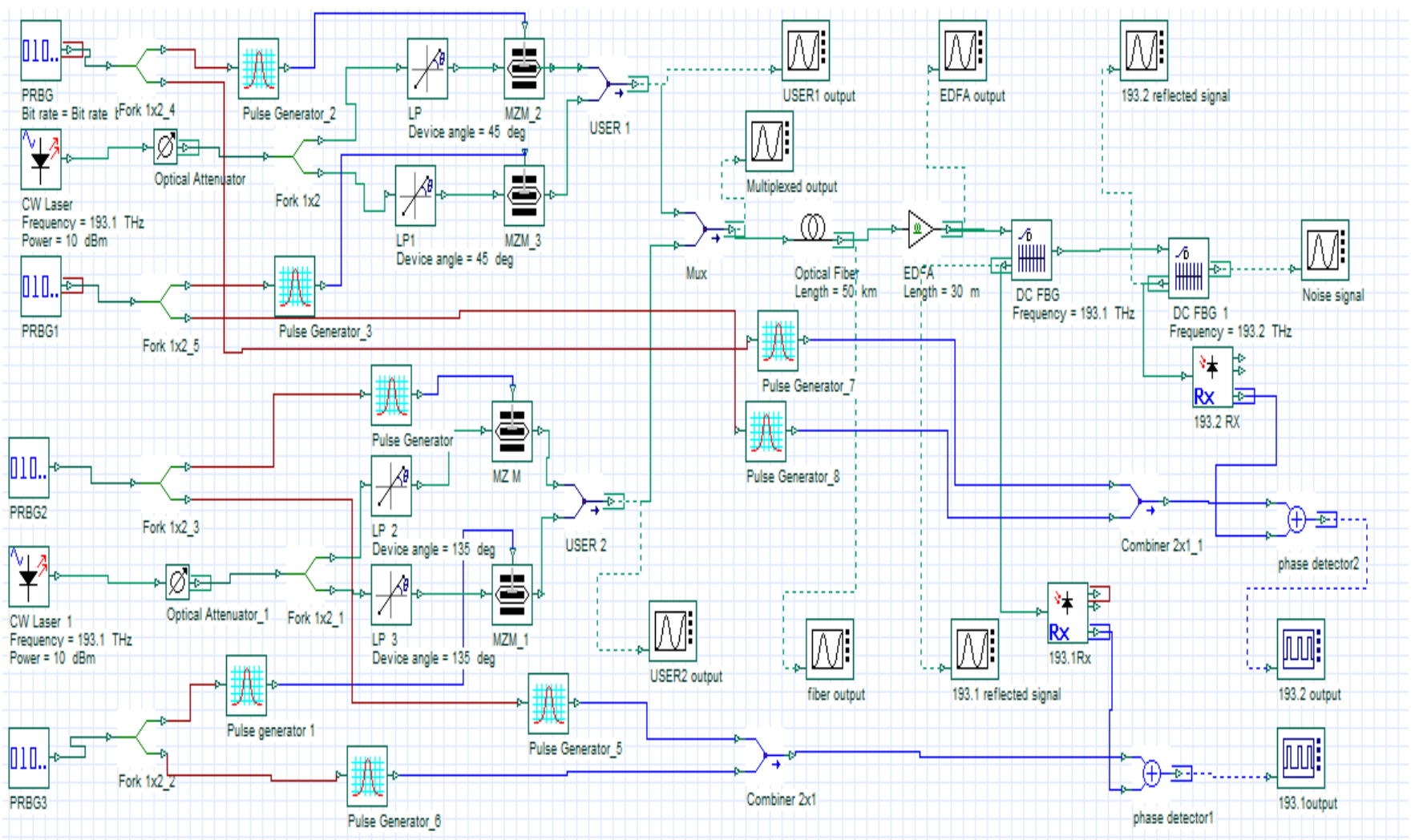

Figure 5: ANITHALIA security model 


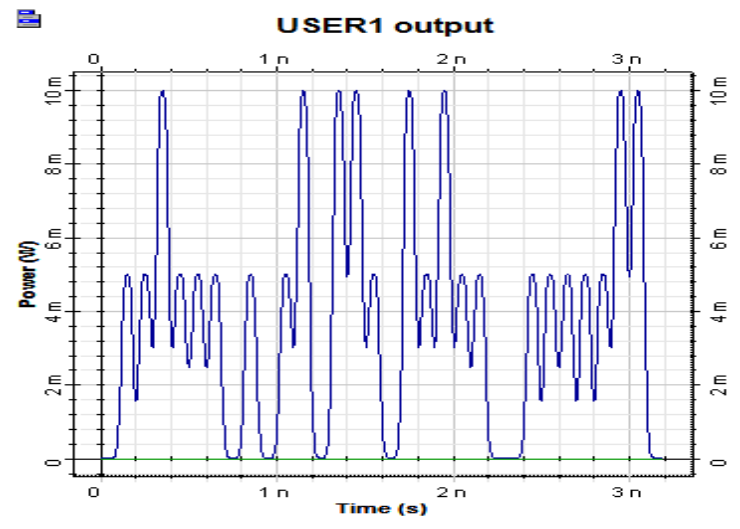

Figure 6.a. Intruder's 193.1 signal.

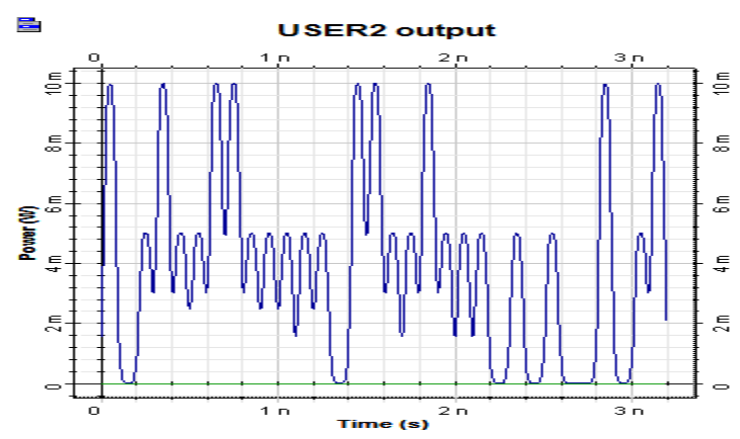

Figure 6.b. Original user's 193.1 signal.

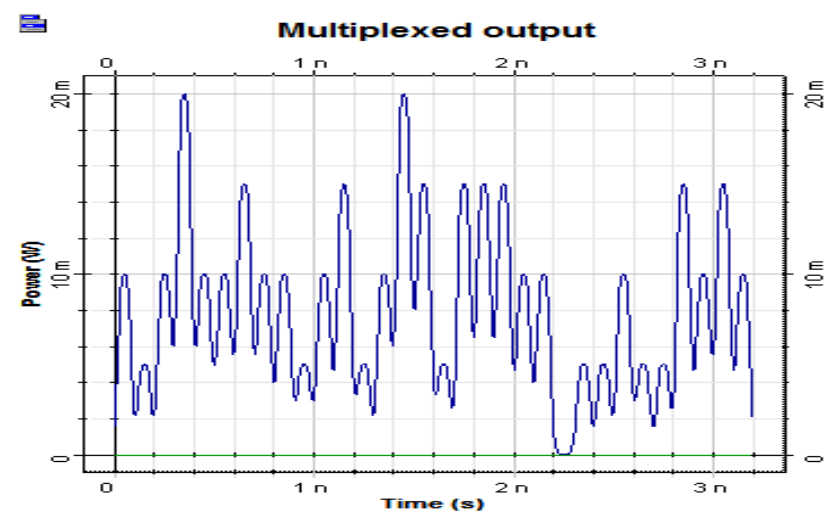

Figure 6.c. Output of the Multiplexed signal. 


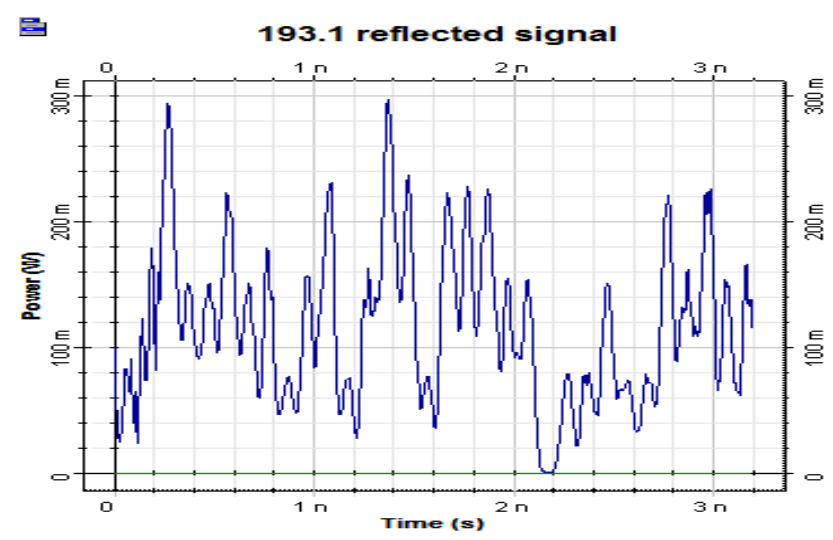

Figure 6.d. Signal reflected by 193.1 filter.

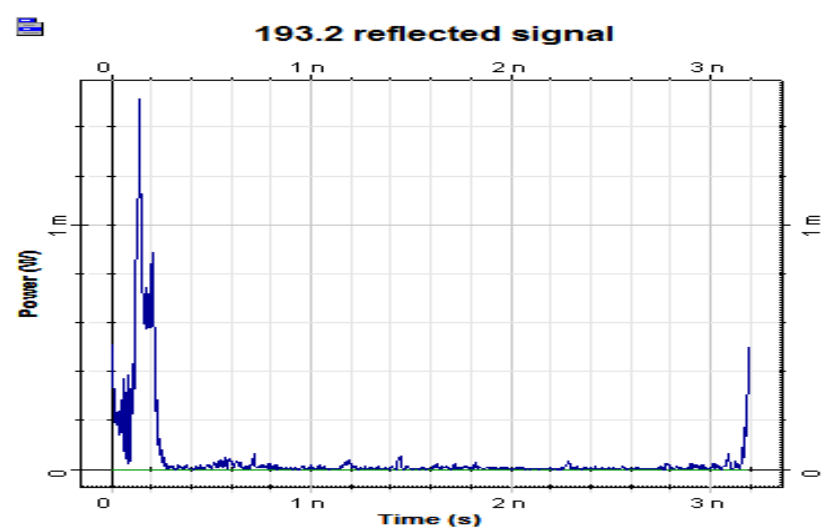

Figure 6.e. Signal reflected by 193.2 filter.

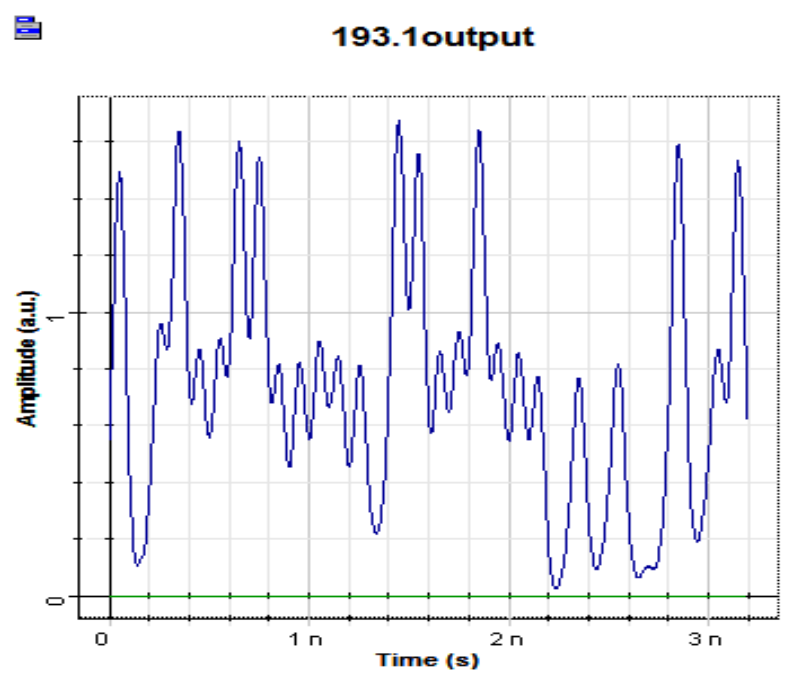

Figure 6.f. Phase detected user's signal. 


\subsection{Conclusion}

Four-wave mixing effects and its possible security issues has been studied and the results have been obtained and analyzed. FWM not only degrades the signals but also imposes a possible security threat on the WDM system. FWM degrades the performance of the WDM system by causing loss of transmitted data which leads to call drops, call interference, and other call related issues. FWM effect can also reduce the signal strength by $60 \%$ and broaden the spectrum of the pulse and these are all alarming factors. The evolution of new pulses due to FWM effect also consumes bandwidth which makes data transmission expensive to telecommunication companies. If an eavesdropper or an intruder gets access to the FWM newly generated signals, the intruder will be able to access part of the transmitted signal thereby compromising the security and confidentiality of the data. The results show that an unequal space channel does better in FWM regime and performs better security wise making WDM promising.

ANITHALIA model with phase detection is designed to detect and separate the adversary's induced signal from the original signal. This system is able to separate the attacker's signal; hence it is termed secured.

\section{References}

Agrawal G. P.” Fiber-optic Communication Systems", John Wiley and Sons, NY, 1997.

Anonymous India hacked BSNL website security affairs, 2015. Retrieved on August 14, 2016 from https://www.securityaffairs.co/wordpress/38315/cyber-crime/anonymous-india-hackedbsnl.html/.

Antwiwaa, A., Okyere-Dankwa, S. and Prince S., "Effect of interaction caused by relative phase, amplitude and spacing on neighboring soliton pulses", in the proceeding of the WCE, UK, 2012.

Goebel B. and Hanik N. "Analytical calculation of the number of four-wave mixing products in optical multichannel communication systems," Technical report, pp. 1-4, Oct., 2008.

K. Inoue, "Four-wave mixing in an optical fiber in the zero-dispersion wavelength region", Journal of Lightwave. Technology, Vol. 10, No.11, pp. 1553-1561, Nov. 1992.

Kaiser W. and Garrett G. "Two-Photon Excitation in CaF2; Eu2+", Physics. Review Letters 7, 229-231 (1961).

Menyuk C. R., Carter G. M., Kath W. L. and Mu R. M. "Dispersion managed solitons and Chirped return to zero: what is the difference? in I. P. Kaminow and T. Li (Eds)", Optical Fiber Telecommunications IVB, Academic Press, pp. 305-328, 2002.

Optical solitons theory and experiments edited J.R. Taylor.

Ramprasad A. and Meenakshi M., "Four Wave Mixing on Dense wavelength division Multiplexing Optical system- a study", Academic Open Internet Journal, Vol. 17, No.1, pp. 1-8, 2006.

Saleh A. and Simmons J. "Technology and architecture to enable the explosive growth of the Internet", IEEE communications magazine, pp.126-132, Jan., 2011.

Song S. "The number of four-wave mixing (FWM) waves in WDM systems and its applications," in Proc. LEOS 2001, San Diego, CA, USA, Nov. 12-13, 2001, Paper TuS4.

Talktalk hack 'affected 157,000 customers', 2015. Retrieved August 15, 2016 from https://www.bbc.co.uk/news/amp/34743185/.

Tkach R., Chraply A., Forghieri F., Gnauck H. and Derosier M. "Four-photon mixing and high-speed WDM systems”, Journal of Lightwave Technology, Vol. 13, No. 5, pp. 841-849, May, 1995.

Vodafone hacked: Almost 2000customer accounts have been accessed by hackers, 2015. Retrieved August 15, 2016 from http://www.cxotoday.com/story/vodafone-cyber-attack-an-eye-opener-totelcos/. 